\title{
Physical Examination Time
}

National Cancer Institute

\section{Source}

National Cancer Institute. Physical Examination Time. NCI Thesaurus. Code C83153.

The time that the physical examination took place. 